\title{
AS CONTRIBUIÇÕES DA INTELIGÊNCIA COMPETITIVA PARA A ESTRATÉGIA NO CONTEXTO DE UMA INSTITUIÇÃO BANCÁRIA
}

\author{
LAS CONTRIBUCIONES DE LA INTELIGENCIA \\ COMPETITIVA PARA LA ESTRATÉGIA EN EL \\ CONTEXTO DE UMA INSTITUCION BANCÁRIA
}

\author{
Maíra Esteves de Carvalhoa \\ Helga Cristina Hedlerb \\ Lillian Maria Araújo de Rezende Alvares ${ }^{\circ}$ \\ Eduardo Amadeu Dutra Moresi ${ }^{d}$ \\ Ana Paula Bernardi da Silvae
}

\begin{abstract}
RESUMO
Introdução: As instituições bancárias brasileiras possuem, em geral, produtos e serviços semelhantes, com pouca distinção entre si. Portanto, a utilização das informações de forma estratégica e inteligente podem determinar o sucesso e a permanência no mercado. A inteligência competitiva (IC) têm se preocupado com o gerenciamento destas informações, estruturando seu processo para o aprimoramento do conhecimento acerca dos seus concorrentes, antecipando-se de forma a conquistar novos mercados e alcançar melhores resultados. Objetivo: Compreender como a IC tem contribuído para o alcance dos objetivos estratégicos de um banco a partir da percepção de gestores, abordando-se a estrutura, recursos pessoais e tecnológicos da IC. Metodologia: A caracterização da visão sistêmica do processo de IC nas instituições financeiras foi realizada através de levantamento bibliográfico, culminando na elaboração de instrumentos de pesquisa. Este foi aplicado para os gestores de um banco de grande porte. Quinze de cinquenta funcionários dos níveis estratégico, tático e operacional da IC participaram da fase de coleta de dados, a qual incluía entrevistas e um grupo focal. Os resultados foram agregados em quatro categorias (visão, ciclo, práticas e desafios) onde cada contempla seus temas. Resultados: A IC foi considerada um elemento chave no processo estratégico ainda que se apresente em estágio inicial. Um dos principais desafios é relacionar os esforços de inteligência com
\end{abstract}

\footnotetext{
a Mestre em Gestão do Conhecimento e Tecnologia da Informação da Universidade Católica de Brasília. E-mail: mairaesteves00@gmail.com

b Doutora em Psicologia Social, do Trabalho e das Organizações pela Universidade de Brasília. Professora da Universidade Católica de Brasília. E-mail: helgachedler@gmail.com

c Doutora em Ciência da Informação pela Universidade de Brasília. Professora da Universidade de Brasília. E-mail: lilian@alvarestech.com

d Doutor em Ciências da Informação pela Universidade de Brasília. Professor da Universidade Católica de Brasília. E-mail: moresi@ucb.br

e Doutora em Engenharia Elétrica pela Universidade de Brasília. Professora da Universidade Católica de Brasília. E-mail: anap.bernardi@gmail.com
} 
Maíra Esteves de Carvalho, Helga Cristina Hedler, Lillian Maria Araújo de Rezende Alvares, Eduardo Amadeu Dutra Moresi, Ana Paula Bernardi da Silva

As contribuições da inteligência competitiva para a estratégia no contexto de uma instituição bancária

os resultados efetivamente alcançados, mensurando assim o seu valor estratégico. Conclusões: Como contribuição teórica deste trabalho foi proposto um processo de IC, que contempla abordagens científicas diferenciadas, sintetizando a dinâmica das etapas ao longo do processo. A contribuição prática se dá através da implantação e estruturação do processo de IC de forma detalhada.

Descritores: Inteligência Competitiva. Tomada de Decisão. Estratégia.

\section{INTRODUÇÃO}

A Inteligência Competitiva - IC tem sido bastante explorada de forma teórica na literatura científica, estando em prevalência aos estudos de cunho empírico. No âmbito das organizações, a IC é considerada uma abordagem essencial nos mais diversos segmentos de atuação. No entanto, estudos empíricos relacionados ao processo de IC em instituições bancárias têm sido pouco relatados na literatura científica (HEPPES; DU TOIT, 2009; LEÃO, 2010; OLIVEIRA; FORTE, 2009; BRESOLIN, 2012). Nesta direção, esta pesquisa buscou contribuir através do estudo dos processos de IC em uma instituição bancária. No que se refere à contribuição teórica, o artigo traz os principais autores e as principais correntes da Inteligência Competitiva aplicada. O trabalho é enriquecido com estudos desenvolvidos no país, voltados para a realidade brasileira.

Nota-se, a partir das publicações revisadas e apresentadas no item revisão de literatura, que as instituições bancárias adotam práticas de inteligência competitiva, no entanto, cabe ainda realizar um esforço para sistematizar tais experiências empíricas destacando qual o impacto desse processo para os resultados estratégicos da empresa, uma vez que pouca ênfase tem sido dada a esse aspecto.

Além disso, apesar da existência de processos internos para a análise de informações em metade das instituições que adotam a IC, as informações geradas e analisadas não retornam a todos os funcionários. Sob o ponto de vista teórico Bresolin (2012) afirma que essa é uma questão a ser tratada. Conhecer os pontos de atenção da organização, compreender o impacto das mudanças e os movimentos do macro ambiente são ações essenciais para que 
Maíra Esteves de Carvalho, Helga Cristina Hedler, Lillian Maria Araújo de Rezende Alvares, Eduardo Amadeu Dutra Moresi, Ana Paula Bernardi da Silva

As contribuições da inteligência competitiva para a estratégia no contexto de uma instituição bancária

todos os funcionários se sintam valorizados, subsidiados e parte integrante da organização.

Sabe-se que as instituições bancárias brasileiras possuem, em geral, produtos e serviços semelhantes, com pouca distinção entre si. Portanto, a utilização das informações de forma estratégica e inteligente pode-se determinar o sucesso e a permanência no mercado. Dentre os processos utilizados para este fim os bancos têm investido na IC e têm se preocupado com o gerenciamento das informações, estruturando seu processo para o aprimoramento do conhecimento acerca dos seus concorrentes, antecipandose de forma a conquistar novos mercados e alcançar melhores resultados (BRESOLIN, 2012).

Nesse sentido, uma questão torna-se pertinente: como a inteligência competitiva tem contribuído para o alcance dos objetivos estratégicos de uma instituição bancária? Para responder a esta questão traçou-se como objetivo principal compreender como ocorre este processo em um banco a partir da percepção de gestores, abordando-se a estrutura, recursos pessoais e tecnológicos da IC.

Esses elementos foram buscados por meio da sistematização de alguns estudos recentes no contexto brasileiro da IC em bancos. Foram subsídios essenciais os estudos de Marin e Poulter (2004), Heppes e Du Toit (2009), Vidigal e Nassif (2012) e Oliveira (2013) a partir dos quais desenvolvemos a pesquisa com o intuito de captar, compreender e descrever as experiências dos principais atores, que atuam em diferentes níveis do processo da IC no Banco.

O artigo foi organizado de forma a esclarecer o conceito de inteligência competitiva, como ocorre seu processo, caracterizar a IC do ponto de vista da pratica diária nas instituições financeiras conforme relatos científicos. Em seguida, apresentaram-se o método, resultados, discussão e considerações finais. 


\section{REVISÃO DE LITERATURA}

Na visão de Heppes e Du Toit (2009), a IC está ganhando espaço e atenção mundialmente, no entanto, há países onde está mais desenvolvida como os Estados Unidos, Austrália, Japão, França e Canadá. No Brasil, a atividade acadêmica de inteligência competitiva teve início na década de 1990, com a criação do Curso de Especialização em Inteligência Competitiva na Universidade Federal do Rio de Janeiro (UFRJ), em parceria com a Université d'Aix-Marseille III. Vale destacar também a fundação da Associação Brasileira dos Analistas de Inteligência Competitiva (ABRAIC) em 2000 (MENDES; MARCIAL; FERNANDES, 2010). A ABRAIC objetiva desenvolver estudos, apoiar a organização de eventos, assessorar a implantação da atividade nas organizações, estimular a pesquisa e reconhecer boas práticas realizando premiações, bem como difundir a IC no país.

A prática de IC tem crescido e ganhado espaço em alguns segmentos empresariais, porém, conforme Millán e Comai (2004), as atividades estão pouco estruturadas e se dão de maneira não sistemática e informal. Da mesma forma, Campelli et al. (2011) afirmam que o problema histórico é que a IC tem sido implantada em grande parte das empresas, de forma desorganizada e assistemática. Heppes e Du Toit (2009) avaliaram de forma geral a evolução do processo nas empresas e concluíram que a maioria está correto. Está com pouca especialização e sofisticação e geralmente se limita ao domínio dos sistemas e serviços de informação, e operam de forma reativa às solicitações dos clientes ou usuários. Entendemos que o autor ressalta que há aplicação de IC pelas empresas, no entanto, não a realizam de forma estratégica e prospectiva/antecipativa.

$\mathrm{Na}$ busca do entendimento do conceito de IC, apresentamos brevemente os critérios do levantamento bibliométrico que sustentaram a pesquisa.

A busca foi realizada em várias bases, com destaque para três principais: Portal da Coordenação de Aperfeiçoamento de Pessoal de Nível 
Maíra Esteves de Carvalho, Helga Cristina Hedler, Lillian Maria Araújo de Rezende Alvares, Eduardo Amadeu Dutra Moresi, Ana Paula Bernardi da Silva

As contribuições da inteligência competitiva para a estratégia no contexto de uma instituição bancária

Superior (CAPES) (http://www.periodicos.capes.gov.br), ScienceDirect (http://www.sciencedirect.com) e Scientific Electronic Library Online (SCIELO) (http://www.scielo.org/). A pesquisa contemplou as seguintes palavras-chave e suas combinações: "competitive intelligence", "financial institutions", "inteligência competitiva" e "instituições financeiras". Além de artigos científicos, outras fontes como livros de autores referenciados, dissertações de mestrado e teses de doutorado, relacionados aos temas também foram consideradas.

Já ao combinar os dois termos anteriores "Competitive Intelligence" e "Financial Institutions" foram apresentados 121 resultados, dentre eles 116 artigos, 33 publicados nos últimos 5 anos, sendo que grande parte das publicações estavam relacionadas às áreas de Tecnologia da Informação e Gestão do Conhecimento. Destes resultados, partindo de uma análise do resumo dos trabalhos, foram selecionados 7 como de maior contribuição para esta pesquisa para aprofundamento na literatura devido à maior afinidade com o tema.

Partindo para a busca de resultados dos termos em português, a combinação dos termos "inteligência competitiva" e "instituições financeiras" foi apresentada em apenas 3 resultados. Dentre estes, apenas um foi selecionado para leitura, pois aborda o planejamento estratégico e cenários nas instituições financeiras em momentos de crise.

Dos resultados encontrados, a amostra é maior nas publicações em inglês. Já em português, o resultado torna-se limitado o que reforça a necessidade de publicações sobre o tema. Portanto, a partir desta busca bibliométrica apresentam-se algumas definições importantes para 0 entendimento da IC nesta pesquisa.

Para Fuld (1994), inteligência é informação analisada que auxilia a tomada de decisão estratégica e tática. Garcia (1997) entende que a inteligência competitiva consiste em um sistema de monitoramento, que pode ser definido como um conjunto de procedimentos para a coleta e análise de informações sobre o macro ambiente, que proporcionam à organização um 
Maíra Esteves de Carvalho, Helga Cristina Hedler, Lillian Maria Araújo de Rezende Alvares, Eduardo Amadeu Dutra Moresi, Ana Paula Bernardi da Silva

As contribuições da inteligência competitiva para a estratégia no contexto de uma instituição bancária

processo de aprendizagem contínuo, voltado ao planejamento e decisões estratégicas.

De acordo com a SCIP (2014), a IC é um programa sistemático e ético de coleta, análise e gerenciamento de informações externas que podem afetar os planos, decisões e operações. A ABRAIC (MENDES; MARCIAL; FERNANDES, 2010) insere também o ambiente interno, a define como o conhecimento do macro ambiente, interno e externo, aplicado aos processos de tomada de decisão nos níveis estratégicos e táticos.

Outros autores (PRESCOTT; MILLER, 2002; CANONGIA et al., 2004; TARAPANOFF, 2006) abordam a IC em termos de definição, caracterização e descrição dos seus processos. Tais autores definem IC com uso de diferentes formas. No entanto, convergem em relação à necessidade de considerar atrelado à IC o conhecimento do ambiente, seu papel em dar suporte à estratégia e subsídio à tomada de decisão.

Na visão de Ganesh, Miree e Prescott (2003), o processo de análise da inteligência competitiva é representado convencionalmente como um ciclo - o ciclo da inteligência, sendo esse o modelo base usado por diversos autores.

Segundo Oliveira (2013), é necessário que a equipe de inteligência responda a duas perguntas principais na primeira etapa: quais são as necessidades de inteligência e quais são seus respectivos usuários. Nessa fase, portanto, é importante a participação e envolvimento não só da equipe de IC, mas também de atores da organização que fazem parte da gestão estratégica e negocial.

Por conseguinte é realizada a coleta de informações, para posteriormente seguir para a fase de análise e tratamento. Nessa etapa, a equipe de IC direciona os seus esforços na identificação das fontes mais confiáveis e relevantes, que podem ser oriundas tanto do ambiente interno quanto externo às organizações.

Marcial (2013) classifica as fontes de informação utilizadas pelos profissionais de inteligência como registradas e não registradas. As informações registradas consistem essencialmente naquelas disponíveis em 
Maíra Esteves de Carvalho, Helga Cristina Hedler, Lillian Maria Araújo de Rezende Alvares, Eduardo Amadeu Dutra Moresi, Ana Paula Bernardi da Silva

As contribuições da inteligência competitiva para a estratégia no contexto de uma instituição bancária

fontes públicas ou até mesmo dentro da própria organização e são obtidas em geral através de buscas na Internet, bases de dados, bibliotecas ou centros de documentações, exemplo, informações registradas jornais, revistas, boletins de imprensa, bases de dados de patentes e periódicos, relatórios e informativos empresariais ou governamentais, bem como panfletos e demais materiais publicitários.

No que se referem às informações não registradas, estas têm como foco principal a fonte humana. Essas informações podem ser obtidas em especial através de técnicas como entrevistas e benchmarking. As informações dessa natureza são bastante significativas, pois o analista de inteligência pode obter por meio delas informações relacionadas ao futuro e à antecipação de movimentos do mercado. Para Marcial (2013), o desenvolvimento e a conservação das redes humanas de informação são fundamentais para a obtenção de informações atualizadas e oportunas.

Tais informações coletadas necessitam passar por validação para este processo a triangulação, que consiste no cruzamento de informações procedentes de várias fontes; e a checagem dos fatos, fase que se antecede ao surgimento da informação (CASTRO; ABREU, 2006). Ainda com relação à etapa de coleta das informações, Abreu et al. (2008) abordam como os relatórios de coleta de informações contribuem para a recuperação rápida das informações e das fontes de coleta.

Na próxima etapa é realizada a análise das informações. Trindade e Rebelo (2005) ressaltam que o analista de inteligência deve transformar as informações coletadas em uma apreciação significativa, completa e confiável. A análise apresenta conclusões sobre o assunto pesquisado, e avalia o impacto da informação para a posição competitiva da organização.

Posteriormente faz-se a disseminação sistemática para as áreas da organização. Assim, após as informações serem coletadas, analisadas e interpretadas, são gerados os principais produtos de IC e disseminados por meio de clippings, relatórios, análise, dossiês e informes. Sobre essa disseminação, pôde-se verificar que a divulgação não é realizada para todos os 
Maíra Esteves de Carvalho, Helga Cristina Hedler, Lillian Maria Araújo de Rezende Alvares, Eduardo Amadeu Dutra Moresi, Ana Paula Bernardi da Silva

As contribuições da inteligência competitiva para a estratégia no contexto de uma instituição bancária

funcionários da empresa, estando restrita a setores e níveis hierárquicos específicos (GOMES; BRAGA, 2006; BRESOLIN, 2012).

$\mathrm{Na}$ abordagem de Bresolin (2012), a inteligência competitiva não deve se limitar a conceder as respostas certas a um decisor em um momento específico, mas deve comunica-las a todos os níveis da organização. Assim, desenvolver um processo de IC com excelência em uma empresa requer alguns requisitos importantes, sendo um deles a promoção de uma cultura organizacional que propicie a troca de informações, vencendo alguns obstáculos como a possível retenção da informação como poder.

O processo de inteligência competitiva, entretanto, não se encerra nesse ponto. Após o percurso dessas quatro etapas como saber se o processo foi efetivo e trouxe resultados estratégicos para a organização? É importante avaliar se o sistema de IC atendeu plenamente às necessidades de inteligência da organização e se necessita de melhorias.

Trindade e Rebelo (2005) incluem uma quinta etapa, que corresponde à fase de avaliação. É a etapa na qual os tomadores de decisão mensuram a satisfação do usuário. Assim, para Castro e Abreu (2006), na avaliação o ciclo se renova ao mesmo tempo que o usuário final retorna ao gerador da informação o nível de conhecimento obtido com a utilização daquela informação, e assim são avaliadas as melhorias necessárias nos métodos e itens utilizados. De forma complementar, Trzeciak, Schenatto e Abreu (2008) destacam que a avaliação do processo de IC deve atuar como insumo de realimentação ao processo. A Figura 1 sintetiza nosso entendimento de como funciona o ciclo de IC. 
Maíra Esteves de Carvalho, Helga Cristina Hedler, Lillian Maria Araújo de Rezende Alvares, Eduardo Amadeu Dutra Moresi, Ana Paula Bernardi da Silva

As contribuições da inteligência competitiva para a estratégia no contexto de uma instituição bancária

\section{Figura 1: Ciclo de inteligência competitiva}

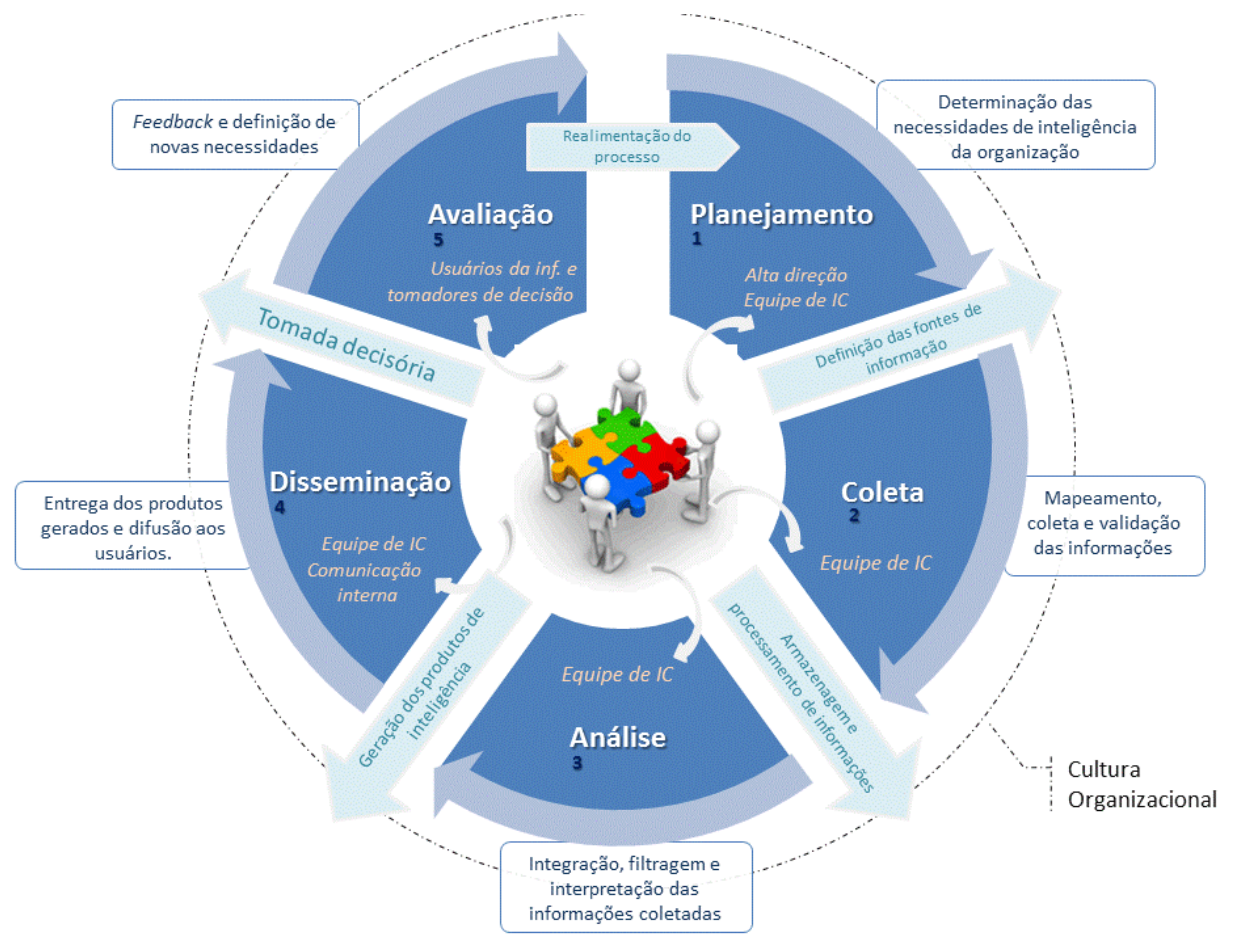

Fonte: os autores.

Para Terra (2008), o sucesso da implementação de IC depende de: a) aderência aos objetivos estratégicos; b) mudança cultural; c) novos processos informais e formais; d) tecnologias de informação e comunicação; e) governança bem articulada e estruturada; f) envolvimento de profissionais altamente qualificados; e g) avaliação de resultados.

Bresolin (2012) afirma que as empresas atualmente dispõem de um excessivo número de informações e o desafio está na tomada de decisões estrategicamente corretas adquirindo vantagem competitiva. Também segundo Santos (2000), de uma forma geral as organizações não têm dificuldade na coleta e armazenamento de informações para a tomada decisória, no entanto, enfrentam o desafio para processar essas informações, de forma que essas se tornem úteis.

Pela própria característica da atividade, as instituições bancárias dispõem de muitas informações sobre seus clientes e competidores, o que representa uma grande vantagem para a implementação de inteligência 
Maíra Esteves de Carvalho, Helga Cristina Hedler, Lillian Maria Araújo de Rezende Alvares, Eduardo Amadeu Dutra Moresi, Ana Paula Bernardi da Silva

As contribuições da inteligência competitiva para a estratégia no contexto de uma instituição bancária

competitiva. Portanto, fica cada vez mais notória a importância do desenvolvimento de processos de inteligência competitiva, de modo a proporcionar um melhor preparo para mudanças inesperadas, visando se antecipar e reagir de forma tempestiva.

\section{ESTUDOS SOBRE IC EM INSTITUIÇÕES BANCÁRIAS}

A mudança nos cenários econômicos é constante e as transformações muitas vezes são de grande impacto, modificando o ambiente concorrencial de empresas e países, desse modo, é preciso se adaptar à realidade imposta por esse ambiente dinâmico. A IC, expressivamente nos últimos anos, tem conquistado maior atenção no setor bancário que busca conquistar melhores condições competitivas e um desempenho superior em seus respectivos mercados consumidores. (BRESONLIN, 2012)

Como bem lembrado por Leão et al. (2010), eventos que impactaram e ensejaram mudanças nas instituições bancárias, dentre outras, como o cenário econômico mundial podem ser recapitulados de modo a compreender essas mudanças, a exemplo da: crise da quebra da bolsa Nova York, a segunda guerra mundial ocorrida de 1939-1945, a guerra fria, a crise do petróleo nos anos 1970, o estouro dos juros internacionais no começo da década de 1980, o surgimento da Internet a partir dos anos 1990, a guerra do Golfo em 1991, a crise de câmbio em 1999, o Bug do milênio em 2000, os atentados terroristas de 11 de setembro de 2001 e a crise empresarial dos Estados Unidos em 2008, refletida nas condições do mercado no mundo todo, inclusive no Brasil. Assim é cada vez mais notória a importância do desenvolvimento de processos de inteligência competitiva nas instituições bancárias, de modo a proporcionar melhor preparo para mudanças inesperadas, e visando se antecipar e reagir de forma tempestiva.

$\mathrm{Na}$ visão de Capuano et al. (2009), os bancos estatais da Alemanha, por exemplo, ocupam um importante papel no controle dos negócios do país e por esse motivo se interessam em proteger seus investimentos. Dessa forma, os bancos têm intencionalmente utilizado seu poder e influência para coletar 
Maíra Esteves de Carvalho, Helga Cristina Hedler, Lillian Maria Araújo de Rezende Alvares, Eduardo Amadeu Dutra Moresi, Ana Paula Bernardi da Silva

As contribuições da inteligência competitiva para a estratégia no contexto de uma instituição bancária

informações privadas sobre empresas estrangeiras e disseminá-las reservadamente às empresas alemãs.

Heppes e Du Toit (2009) avaliaram o grau de maturidade do processo de IC em um banco de varejo Sul Africano, estabelecendo características-chave dos diferentes graus de maturidade. Na visão desses autores, as práticas de IC vêm se destacando na África do Sul, ainda que não estejam nos níveis de países como os EUA, Austrália, Japão, França e Canadá. Assim foi préselecionado um grupo de participantes desse banco e aplicados questionários e entrevistas pessoais. As variáveis estudadas foram:

a) as informações-chave necessárias aos usuários de IC (Key Intelligence Needs - KINs);

b) as capacidades de entrega e os resultados de IC;

c) os produtos gerados;

d) a relação com a gestão;

e) as fontes de informação;

f) a qualificação da equipe de IC e há quanto tempo o processo está implantado.

Os resultados dessa pesquisa também oferecem algumas recomendações de como os bancos de varejo podem evoluir do grau médio de maturidade para o que eles chamam de um nível de classe mundial, que seria um estágio mais evoluído de maturidade. Assim, compreendem a evolução básica de um processo de IC a partir de estágios inicial, médio e evoluído. $\mathrm{O}$ estágio, ou fase inicial, consiste naquele que fornece apenas os fatos e o que está acontecendo no macro ambiente, iniciando a criação de consciência de IC na organização. Na fase de média capacidade de IC, já se identificam as tendências e implicações a partir das informações coletadas, dentro de uma parceria com os usuários de IC. Na fase mais evoluída de capacidade de IC nível de classe mundial - o processo de IC é considerado um elemento-chave da estratégia da empresa.

Desse modo, avaliando o contexto das organizações do sistema bancário, e a partir da pesquisa aplicada, concluiu-se que para o processo de 
Maíra Esteves de Carvalho, Helga Cristina Hedler, Lillian Maria Araújo de Rezende Alvares, Eduardo Amadeu Dutra Moresi, Ana Paula Bernardi da Silva

As contribuições da inteligência competitiva para a estratégia no contexto de uma instituição bancária

IC evoluir de uma ação ou processo do tipo fornecer apenas os fatos (postura reativa), para ser um elemento-chave da estratégia da empresa (postura proativa), segundo Heppes e Du Toit (2009) são necessários os seguintes passos:

a) o processo de IC deve ser dotado dos recursos necessários para viabilizar sua implementação, isso inclui a designação de uma equipe específica em tempo integral e a aplicação de softwares específicos de IC;

b) auxiliar a alta administração no desenvolvimento e revisão do conjunto das principais necessidades de Inteligência (KINs), assim como deve desenvolver e fornecer informações sobre as tendências e implicações dessas necessidades, por meio de recursos e habilidades analíticas;

c) desenvolver e fornecer sinais de alerta antecipado, através do mapeamento das áreas de alto risco (KINs), e assim construir e monitorar os indicadores, emitindo alertas de como estes riscos podem se desdobrar e seus impactos;

d) desenvolver as competências dos analistas de IC para a análise e síntese de informações, de modo que sejam fornecidos insights significativos, para converter as informações em um subsídio valioso para a tomada de decisão e ação.

Com isso, pode-se afirmar que a aplicação da IC de forma efetiva aumenta a vantagem competitiva de uma empresa, a partir de uma melhor compreensão do ambiente interno e externo da empresa, levando à melhoria da gestão estratégica.

Avaliando o cenário financeiro brasileiro, Bresolin (2012) selecionou uma amostra de 50 bancos públicos e privados cadastrados na Federação Brasileira de Bancos (FEBRABAN), destacando-se os seis maiores e mais representativos. Assim levantou as práticas de inteligência competitiva aplicando um questionário com 20 perguntas fechadas, sobre a estrutura funcional, objetivos, motivos, fontes, processos, usos, papel da $\mathrm{TI}$ e 
Maíra Esteves de Carvalho, Helga Cristina Hedler, Lillian Maria Araújo de Rezende Alvares, Eduardo Amadeu Dutra Moresi, Ana Paula Bernardi da Silva

As contribuições da inteligência competitiva para a estratégia no contexto de uma instituição bancária

disciplinamento ético da IC nas organizações pesquisadas. Das 50 instituições bancárias abordadas, 15 responderam (30\% de retorno efetivo). Alguns achados importantes puderam ser observados:

a) apenas 3 instituições bancárias possuíam um setor independente destinado à área de inteligência competitiva;

b) as instituições investem a maior ou toda parte do tempo nos estudos de cenários já existentes e realizam poucas pesquisas para intuir sobre o futuro, o que demonstra que a maioria não utiliza a inteligência antecipativa;

c) as equipes dedicadas ao processo de IC nas organizações em sua maioria são representadas por 6 até 10 pessoas;

d) as principais fontes de informação utilizadas são a Internet, pesquisas de satisfação de clientes e pesquisas de clima organizacional junto aos funcionários, sendo o foco de atenção principal dessa etapa os produtos e serviços oferecidos pela concorrência. Além disso, a coleta das informações é realizada diariamente, através de meios e aplicativos específicos;

e) a maioria das instituições analisa informações por meio de análise de cenários e entorno competitivo, e a distribuição das informações é restrita a alguns setores específicos, como Conselho de Administração, Marketing e Diretorias Estratégicas e Executivas.

Pode-se verificar nessa pesquisa que todas as instituições bancárias pesquisadas já adotam práticas de inteligência competitiva. Embora o setor seja altamente informatizado, normatizado e de alto nível de concorrência, grande parte das instituições estão em estágio inicial de implantação de setores de IC independentes. Além disso, apesar da existência de processos internos para a análise de informações em metade das instituições, as informações geradas e analisadas não retornam a todos os funcionários (BRESOLIN, 2012). 
Maíra Esteves de Carvalho, Helga Cristina Hedler, Lillian Maria Araújo de Rezende Alvares, Eduardo Amadeu Dutra Moresi, Ana Paula Bernardi da Silva

As contribuições da inteligência competitiva para a estratégia no contexto de uma instituição bancária

Os estudos encontrados sobre o tema demonstram que os bancos brasileiros já dispõem de práticas de IC, mas diferem em relação aos estágios e graus de maturidade do processo em cada instituição.

\section{METODOLOGIA}

Visando analisar o funcionamento do processo de inteligência competitiva na instituição bancária e suas contribuições para a estratégia, conforme a percepção dos atores-chave que atuam em diferentes níveis desse processo realizou-se uma pesquisa exploratória, qualitativa e aplicada (CRESWELL, 2010). Assim, realizamos duas etapas: na primeira um levantamento bibliográfico, com o objetivo de obter uma visão sistêmica da área e elaborar o instrumento de pesquisa; na segunda a pesquisa de campo.

A instituição bancária de grande porte e entre as cinco maiores do Brasil, denominada Banco Kanopus para preservar o sigilo, possui práticas de inteligência competitiva, conta com um processo de IC institucionalizado e tem investido progressivamente nessa área. $\mathrm{Na}$ última revisão do Planejamento Estratégico do Kanopus em 2011, foram criadas na estrutura organizacional áreas/departamentos exclusivos para o processo de inteligência competitiva, visando subsidiar o alcance dos objetivos estratégicos da organização. $O$ Banco Kanopus conta ainda com áreas estratégicas e táticas, o Conselho Diretor e a Presidência. O processo de IC é descentralizado, portanto a organização dispõe de áreas de inteligência distribuídas em diferentes unidades dentro sede do Banco onde foi realizada a pesquisa

Devido à natureza da pesquisa, a amostra foi intencional e os participantes da pesquisa os colaboradores diretamente envolvidos e ocupam funções de gestores estratégicos, táticos, analistas e técnicos. Estes dois últimos foram incluídos pois desenvolvem atividades diretamente relacionadas ao processo de inteligência competitiva nas Áreas Táticas e Estratégica de IC.

Essas áreas contam com um total de 50 pessoas, destas, 12 participaram voluntariamente, sendo esclarecidos os objetivos da pesquisa e assegurado o sigilo dos participantes. 
Maíra Esteves de Carvalho, Helga Cristina Hedler, Lillian Maria Araújo de Rezende Alvares, Eduardo Amadeu Dutra Moresi, Ana Paula Bernardi da Silva

As contribuições da inteligência competitiva para a estratégia no contexto de uma instituição bancária

A coleta de dados se deu a partir da realização de entrevistas e grupo focal. O grupo focal foi realizado na sede da organização, durou cerca de $1 \mathrm{~h}$ e $30 \mathrm{~min}$ e contou com 7 participantes. As entrevistas foram realizadas nos meses de julho e agosto e o grupo focal foi realizado no mês de setembro de 2014.

Foram aplicados dois tipos de entrevistas semiestruturadas, uma para gestores do nível estratégico e outra para gestores do nível intermediário/tático. As entrevistas com os gestores duraram em média 40 minutos, foram realizadas face a face, posteriormente foram transcritas para análise.

As questões tiveram como base principal Marin e Poulter (2004), Heppes e Du Toit (2009), Vidigal e Nassif (2012), Oliveira (2013) e Castro e Abreu (2006).

O roteiro de entrevista aplicado aos gestores de nível estratégico buscou uma visão mais abrangente do processo. Já o roteiro voltado para os gestores do nível intermediário/tático, explorou as práticas do processo na organização. As perguntas foram dividas em blocos, seguindo três categorias centrais conforme Vidigal e Nassif (2012):

a) Estrutura: localização hierárquica, centralização ou descentralização;

b) Processo Prático de IC: como acontece o planejamento, coleta, análise, disseminação e avaliação;

c) Recursos Pessoais e Tecnológicos: quantidade de pessoas, características e perfis dos profissionais, técnicas e softwares utilizados.

Para o roteiro de Grupo Focal, foram elaboradas questões voltadas aos empregados que trabalham diretamente com o processo de IC no nível de staff, ou seja, operacionalizando e executando a atividade. Os dados foram analisados, segundo a análise de conteúdo categorial temática, proposta por Bardin (2011).

\section{RESULTADOS E DISCUSSÃO}

Os resultados foram agregados em quatro categorias comportando os respectivos temas. Cada tema possui pelo menos duas frequências, conforme 
Maíra Esteves de Carvalho, Helga Cristina Hedler, Lillian Maria Araújo de Rezende Alvares, Eduardo Amadeu Dutra Moresi, Ana Paula Bernardi da Silva

As contribuições da inteligência competitiva para a estratégia no contexto de uma instituição bancária

recomenda Bardin (2011). O Quadro 1 apresenta as categorias de análise, temas e frequências.

\subsection{Categoria: Visão}

Representa as percepções dos participantes em relação ao processo e estrutura de inteligência competitiva na instituição, a concepção do que é IC e seus objetivos.

Referente ao tema "Estrutura", que trata da posição hierárquica das áreas de IC no organograma e a descrição dos aspectos físicos, humanos e financeiros, a maioria dos participantes está satisfeita e relata que ela empodera, no sentido de que atuar nessa área da instituição concede status e que as ações desenvolvidas na área são valorizadas. Entende-se que isto é um elemento favorável para o funcionamento da mesma na instituição. Observa-se que as áreas táticas de inteligência estão vinculadas diretamente às áreas do Conselho Diretor, portanto o contato direto com o tomador de decisão de mais alto nível, propicia acesso aos tomadores de decisão e facilidade de trânsito e relacionamento entre as áreas. Oliveira (2013) investigou o processo de IC em 66 organizações vinculadas a 15 principais setores da economia brasileira e constatou que todas elas dispõem de unidades estruturadas dedicadas exclusivamente ao processo. $O$ fato de o Banco Kanopus também possuir estrutura para IC evidencia que está alinhado às novas tendências, mesmo que o processo ainda seja incipiente e apresente deficiências tecnológicas e equipe não exclusiva.

Quadro 1: Visão geral dos resultados

\begin{tabular}{|c|c|c|c|}
\hline № & Categoria & Temas & Frequência \\
\hline \multirow{3}{*}{1} & \multirow{3}{*}{ Visão } & Objetivos & 19 \\
\cline { 3 - 3 } & & Estrutura & 16 \\
\cline { 3 - 4 } & & Conceitos & 4 \\
\hline \multirow{3}{*}{2} & \multirow{3}{*}{ Ciclo } & Total & 38 \\
\cline { 3 - 4 } & & Disseminação & 23 \\
\cline { 3 - 4 } & & Coleta & 9 \\
\cline { 3 - 4 } & & Análise & 7 \\
\cline { 3 - 4 } & & Avaliação & 5 \\
\cline { 3 - 4 } & & Planejamento & 3 \\
\hline
\end{tabular}

Inf. Inf Londrina, v. 23, n. 1, p. 225 - 252, jan./abr. 2018 http://www.uel.br/revistas/informacao/ 
Maíra Esteves de Carvalho, Helga Cristina Hedler, Lillian Maria Araújo de Rezende Alvares, Eduardo Amadeu Dutra Moresi, Ana Paula Bernardi da Silva

As contribuições da inteligência competitiva para a estratégia no contexto de uma instituição bancária

\begin{tabular}{|c|c|c|c|}
\hline & \multicolumn{2}{|c|}{ Total } & $\mathbf{4 7}$ \\
\hline \multirow{4}{*}{3} & \multirow{4}{*}{ Práticas } & Atividades desenvolvidas & 9 \\
\cline { 3 - 4 } & & Incentivos à capacitação & 6 \\
\cline { 3 - 4 } & & $\begin{array}{c}\text { Relevância e } \\
\text { aplicabilidade }\end{array}$ & 4 \\
& & Patrocínio dos gestores & 2 \\
\cline { 3 - 4 } & & Total & $\mathbf{2 1}$ \\
\hline \multirow{3}{*}{4} & \multirow{2}{*}{ Desafios } & Cultura & 22 \\
\cline { 3 - 4 } & & Papel da IC & 8 \\
\cline { 3 - 4 } & & \multicolumn{2}{|c|}{ Total } \\
\hline
\end{tabular}

Fonte: dados do trabalho.

Nota-se também que há uma clara distinção nos níveis de desenvolvimento em que cada área se encontra, sendo a Área Tática 3 (total de 7 áreas táticas) considerada referência e tida como a de nível mais avançado em IC na organização. Exemplo, “[...] a IC no banco ainda é embrionária em algumas áreas... A área 3 está numa maturidade melhor...[sic]" (participante 6).

O tema "Conceito" considera o ponto de vista dos participantes sobre o que é IC segundo a sua percepção. A maioria dos atores do grupo focal relatou entendimentos restritos em relação à informação. Os "Objetivos" se referem à percepção dos participantes quanto à finalidade do processo. Foram apresentados os entendimentos: monitorar o ambiente externo; estudar cenários; prover inteligência; viabilizar a inovação; tornar processos eficientes e otimizados; produzir visão sistêmica; dentre outros. No entanto, houve uma predominância nas respostas para as finalidades de: antecipar e subsidiar decisões (9 frequências); e prestar recomendações para a reformulação de estratégia de negócio (10 frequências). Isso revela que há clareza quanto à finalidade de IC para a organização, e confirma a abordagem de Popa e Cucui (2009) e Campelli et al., (2011) para os quais o objetivo é responder prontamente às situações que se apresentam, proporcionando um melhor suporte à tomada de decisões e contribuindo para o alinhamento estratégico. 


\subsection{Categoria: Ciclo}

Puderam-se comparar as atividades de inteligência realizadas com 0 Ciclo descrito na literatura. Nota-se que o Banco Kanopus desenvolve todas as etapas, no entanto, cada departamento executa essas atividades sem padronização nas metodologias. Ou seja, cada departamento ligado às atividades de IC exerce suas atividades de forma diferente em relação as demais, usando diferentes formas de coleta, análise, avaliação e ferramentas de suporte à atividade e produtos gerados.

"Planejamento" refere-se à primeira etapa e trata das diretrizes, planejamento e direção das atividades. A maioria dos participantes informou que as necessidades de informação são definidas pelos gestores e alta direção: "[...] quem me dá a diretriz do que eu devo olhar lá fora é o vicepresidente... [sic]" (participante 3 ).

A "Coleta" se refere à obtenção de informações. Observou-se que todas as áreas realizam o monitoramento de mercado, em diferentes amplitudes, algumas áreas o realizam concentrando-se em informações de um segmento específico, e outras monitoram diversos segmentos e em âmbito mundial.

Quanto ao suporte tecnológico para a coleta de informações, nota-se que há diferenças nos tipos de suporte disponíveis entre as áreas. Algumas não possuem software específico, outras contratam/terceirizam e há quem desenvolva internamente de acordo com sua necessidade. Exemplo: "[...] estamos criando um software pra coleta de informação, um aplicativo para monitorar a concorrência... [sic]" (participante 5).

Como um dos meios de coleta de dados, foi apontada por alguns respondentes a Rede de Inteligência Competitiva (RIC), que consiste em uma rede formada por funcionários que já trabalharam em outros bancos. O objetivo é obter informações subjetivas e privilegiadas sobre os concorrentes a partir de ex-funcionários. Ela configura a utilização de fonte de informações não registradas, como foco principal a fonte humana. A ampliação dessas redes informais defendida unanimemente pelos participantes como de suma importância. 
Maíra Esteves de Carvalho, Helga Cristina Hedler, Lillian Maria Araújo de Rezende Alvares, Eduardo Amadeu Dutra Moresi, Ana Paula Bernardi da Silva

As contribuições da inteligência competitiva para a estratégia no contexto de uma instituição bancária

A "Análise" se refere à terceira etapa do ciclo. Conforme os participantes, as áreas táticas de IC trabalham com diferentes metodologias. Um fator comum observado foi a realização de cruzamento de dados externos com internos. Exemplo: "[...] lá na equipe, eu faço um trabalho de demonstração de resultados internos e os outros colegas fazem um olhar externo, mercado, produtos que tem share para alavancar... [sic]" (participante 12).

A "Disseminação" se refere ao acesso aos produtos gerados no processo. São eles: informativos gerenciais, relatórios executivos, boletins, pesquisas, sínteses de indicadores da estatística bancária, alertas de mercado, relatórios e estudos comentados, dentre outros. A periodicidade varia entre diário, semanal e mensal, podendo também ser pontual/esporádica. A principal forma de disseminação é a intranet, também via redes sociais internas e reuniões.

Os produtos em geral são disponibilizados a todos os funcionários, mas em alguns casos são de acesso restrito à alta direção e gestores, o que corrobora com Marin e Poulter (2004), Vidigal e Nassif (2012) e Oliveira (2013). Exemplo: "[...] quando falamos sobre dados do mercado, fica disponível para todos. Quando a informação é mais sensível, fica mais restrita para gestores e alta direção... [sic]"(participante 3).

A "Avaliação" é a última etapa do ciclo. Nota-se que é a etapa menos executada. Não há um modelo padrão de avaliação, em alguns casos acontece por meio de feedbacks dos usuários, e em outros, sequer é realizada, ou seja, ainda é assistemática. Trzeciak, Schenatto e Abreu (2008) destacam a importância desta etapa como insumo de realimentação.

Em uma das áreas táticas foi criado um sistema próprio de avaliação baseado em indicadores. "Estamos começando a implantar...medimos $o$ resultado da Área Tática 3 por três indicadores: produtividade, que mede a quantidade de produtos e abrangência; qualidade, em forma, conteúdo, timing certo, se ajudou a tomar uma decisão; e relevância, que avalia se o que está sendo proposto tá sendo implantado. [sic]"(participante 5). 
Maíra Esteves de Carvalho, Helga Cristina Hedler, Lillian Maria Araújo de Rezende Alvares, Eduardo Amadeu Dutra Moresi, Ana Paula Bernardi da Silva

As contribuições da inteligência competitiva para a estratégia no contexto de uma instituição bancária

O modelo de avaliação proposto pode ser considerado como uma forma de mensuração do valor estratégico da IC. Essa proposta atende a recomendação de Castro e Abreu (2006), pela qual o usuário final retorna ao produtor da informação o nível de conhecimento obtido com a utilização daquela informação, e assim são avaliadas as melhorias necessárias nos métodos e itens utilizados em cada etapa do ciclo.

\subsection{Categoria: Práticas}

Nesta categoria deu-se enfoque à realidade da empresa. O tema "Atividades desenvolvidas" representa o que é realizado pelos membros das equipes de IC. Nota-se que as áreas Táticas 2 e 3 dispõem de processo estruturado baseado no ciclo adotado na pesquisa (Figura 1). Verificou-se também clareza na divisão dos papéis, na qual cada profissional possui habilidades e conhecimentos específicos para o exercício de suas atividades.

Entretanto, foi relatado por alguns participantes que a equipe de IC atende demandas que não estão relacionados à IC, e em alguns casos as atividades se restringem ao simples fornecimento de informações. Essas situações podem se constituir em desafios.

O "patrocínio dos gestores" representa a percepção dos participantes ao uso mais efetivo do potencial dessa equipe e de seus resultados, pois referem ser este o fator de sucesso da área e das atividades desenvolvidas. Entretanto, pôde-se inferir que o grau de patrocínio do gestor, depende da relevância que essa área possui na empresa, em termos de impacto político e financeiro, e também do perfil e estilo do líder. Na fala do Participante 1, observa-se a relevância dada à área, pelo presidente, mas sabe-se que a mudança de cultura organizacional não acontece em curto prazo, a internalização do processo por parte dos funcionários é um processo de médio/longo prazo, “[...] hoje temos um perfil de presidente muito ligado à inteligência, inovador, parceiro. Mas é preciso que isso seja trazido pra veia mesmo, institucionalizado. Esse é o primeiro passo para a mudança de cultura. [sic]"(participante 1). 
Maíra Esteves de Carvalho, Helga Cristina Hedler, Lillian Maria Araújo de Rezende Alvares, Eduardo Amadeu Dutra Moresi, Ana Paula Bernardi da Silva

As contribuições da inteligência competitiva para a estratégia no contexto de uma instituição bancária

Em relação aos "Incentivos à capacitação", verificou-se que há apoio para capacitação, mas não de forma equitativa, pois foi relatado por parte dos profissionais de nível de staff, de que esses incentivos e capacitações estão focados apenas em gestores. Isso pode ser verificado nas respostas do Participante 3, ocupante de função gerencial tática, e do Participante 9, ocupante de função técnica operacional. Exemplo: "Sim, sempre. Eles mandam pra gente uma lista de curso e etc. [sic]" (participante 3); "Não é só a Área Tática 3 que precisará desse patrocínio financeiro, nós também precisamos de capacitação e treinamento. [sic]"(participante 9).

Quanto ao tema "Relevância e aplicabilidade", observa-se que os respondentes são unanimes em ressaltar a importância em relação ao papel da IC e do seu trabalho nela desenvolvido, e isso se reflete em motivação dos envolvidos.

\subsection{Categoria: Desafios}

Conforme participantes, a cultura conservadora é o principal desafio. $O$ tema "Cultura" teve a maior frequência (22) na categoria. Para o desenvolvimento de um processo de IC com excelência é necessária à promoção de uma cultura organizacional que propicie a troca, conforme Bresolin (2012), a cultura deve ser favorável ao compartilhamento de informações e à busca do conhecimento. Foram apontados também: conservadorismo, aversão ao risco, aversão à mudança, burocracia, visão de curto prazo, retenção de conhecimento e não compartilhamento.

O "Papel da IC" se refere à compreensão do papel do processo de inteligência e da equipe. Um dos principais desafios apontados seria a dificuldade de compreensão, por parte dos colaboradores e áreas da empresa, do real objetivo da IC para o Banco Kanopus e o papel das equipes. A falta de clareza faz com que o processo não seja valorizado e reconhecido; além dos colaboradores terem suas atividades desviadas. Outro desafio citado é a integração das áreas de IC. Relatou-se falta de conexão e relacionamento 
Maíra Esteves de Carvalho, Helga Cristina Hedler, Lillian Maria Araújo de Rezende Alvares, Eduardo Amadeu Dutra Moresi, Ana Paula Bernardi da Silva

As contribuições da inteligência competitiva para a estratégia no contexto de uma instituição bancária

entre as áreas táticas de IC, sendo este um fator importante para a integração das equipes e compartilhamento de práticas e experiências.

\section{CONSIDERAÇÕES FINAIS}

Os resultados revelam que as contribuições de IC estão relacionadas ao subsidio, construção e a reformulação da estratégia organizacional no contexto do Banco Kanopus conforme os atores envolvidos relataram. A IC é um elemento-chave no processo estratégico, auxiliando na definição de metas, revisão dos objetivos estratégicos empresariais, impulsionando mudanças organizacionais e apoiando o processo de inovação. Isto também foi corroborado pela existência formal/física de uma área estruturada para este fim. Os atores-chave envolvidos atribuem significado, relevância e aplicabilidade com relação ao trabalho de IC realizado no banco Kanopus. Entretanto há ainda um caminho a ser percorrido para o ajuste desse processo na organização de forma que seja mais valorizado e reconhecido.

Destaca-se a importância de uma estruturação do processo de inteligência em âmbito corporativo, unificando as atuações das áreas táticas de IC, a capacitação das equipes, o investimento em recursos tecnológicos para suporte à atividade, e, sobretudo, a mudança cultural da organização, como dimensões mais alinhadas à IC, como menor aversão ao risco, por exemplo, aspecto esse também enfatizado na literatura.

A aplicação da IC no banco Kanopus se alinha em muitos aspectos ao que vem sido aplicado em empresas que implantaram o processo. Apesar de não existir um modelo padrão de funcionamento, nota-se uma semelhança nas técnicas e métodos utilizados na prática Porém vê-se que algumas práticas encontradas na pesquisa refletem especificidades do setor bancário, como a utilização de redes informais de inteligência competitiva formada por exfuncionários dos concorrentes.

Dentre as limitações desta pesquisa está à impossibilidade de divulgação de alguns dados e documentos utilizados na pesquisa devido ao sigilo das informações, por serem de caráter competitivo e a disponibilização 
Maíra Esteves de Carvalho, Helga Cristina Hedler, Lillian Maria Araújo de Rezende Alvares, Eduardo Amadeu Dutra Moresi, Ana Paula Bernardi da Silva

As contribuições da inteligência competitiva para a estratégia no contexto de uma instituição bancária

deles aos concorrentes do banco, poderia ser uma ameaça. Além disso, a quantidade de respondentes da pesquisa (12) pode ser considerada limitada, perfazendo um número restrito de observações, e requerendo validações das inferências levantadas em outras empresas e com maior quantidade de respondentes.

Esta pesquisa traz contribuições teóricas ao propor um modelo de ciclo ou processo de inteligência competitiva que sintetiza a dinâmica das etapas ao longo do processo de IC. Esse modelo abarca abordagens científicas diferenciadas e visa trazer uma visão consolidada do funcionamento da IC em uma organização. Sob o enfoque prático, espera-se ter contribuído com a implantação e estruturação do processo de inteligência competitiva nas empresas, ao apresentar o detalhamento das atividades desenvolvidas em uma área de IC e as percepções de atores-chave quanto aos desafios e sugestões para uma boa implantação e o alcance dos resultados almejados.

Algumas pesquisas podem ser desenvolvidas a partir deste estudo. Uma delas é a construção de um modelo de avaliação do grau de maturidade das empresas em relação ao processo de IC. Outra possibilidade é propor um modelo de mensuração do valor estratégico da IC, que possibilite a comparação do nível de eficiência de IC entre os bancos, por exemplo. Também é importante investigar os desafios da implantação de IC em organizações do setor público, considerando a característica sólida e não tão competitiva desse setor.

Cabe ressaltar também que neste trabalho a análise dos dados das entrevistas e do grupo focal foi realizada de forma conjunta, no entanto, para trabalhos futuros o procedimento pode ser realizado de forma segregada, analisando-se primeiro os dados das entrevistas e depois os dados do grupo focal, o que gerará uma análise individualizada de cada tipo de instrumento de pesquisa que pode acarretar na obtenção de resultados distintos.

A inteligência competitiva tem se tornado um importante campo de pesquisa atualmente e nota-se que muitas questões ainda necessitam de ser mais bem 
Maíra Esteves de Carvalho, Helga Cristina Hedler, Lillian Maria Araújo de Rezende Alvares, Eduardo Amadeu Dutra Moresi, Ana Paula Bernardi da Silva

As contribuições da inteligência competitiva para a estratégia no contexto de uma instituição bancária

exploradas pelos pesquisadores contemporâneos, em especial no contexto das instituições bancárias brasileiras.

\section{REFERÊNCIAS}

ABREU, A. F. et al. Inteligência competitiva. In: CORAL, E.; OGLIARI, A. \& ABREU, A. F. Gestão Integrada de inovação: estratégia, organização e desenvolvimento de produtos. São Paulo: Atlas, 2008. Cap. 6, p. 113-135.

ASSOCIAÇÃO BRASILEIRA DOS ANALISTAS DE INTELIGÊNCIA COMPETITIVA - ABRAIC. História da IC no Brasil. Disponível em: < http://abraic.org.br/inf.php?idAtual=1\&idTela=5>. Acesso em: 05 abr. 2014.

BARDIN, L. Análise de conteúdo. São Paulo: Edições 70, 2011.

BRESOLIN, L. K. A inteligência nas instituições financeiras brasileiras. 2012. 62f., il. TCC (Especialização em Administração) - Universidade Federal do Rio Grande do Sul, Porto Alegre, 2012.

CAMPELLI, M. G. R.; BARBEJAT, M. E. R. P.; CASAROTTO FILHO, N.; N. M. Perspectivas da inteligência competitiva no Brasil. In: CONGRESSO BRASILEIRO DE EDUCAÇÃO EM ENGENHARIA, 34., 2011, Blumenau/SC. Anais...Blumenau: COBENGE, 2011. Disponível em: $<$ http://www.abenge.org.br/cobenge/arquivos/8/sessoestec/art1706.pdf $>$. Acesso em: 13 mar. 2014.

CANONGIA, C. et al. Foresight, inteligência competitiva e gestão do conhecimento: instrumentos para a gestão da inovação. Gestão \& Produção, v. 11, n. 2, p. 231-238, 2004. Disponível em: $<$ http://www.scielo.br/pdf/\%0D/gp/v11n2/a09v11n2.pdf $\geq$. Acesso em: 07 nov. 2013.

CAPUANO, E. A. et al. Inteligência competitiva e suas conexões epistemológicas com gestão da informação e do conhecimento. Ciência da Informação, Brasília, v 38, n. 2, p. 19-34, 2009.

CASTRO, J. M.; ABREU, P. G.F. Influência da inteligência competitiva em processos decisórios no ciclo de vida das organizações. Ciência da Informação, v. 35, n. 3, p. 15-29, 2006.

CRESWELL, J. W. Projeto de Pesquisa: Métodos qualitativo, quantitativo e misto. Porto Alegre: Artmed, 2010. 
Maíra Esteves de Carvalho, Helga Cristina Hedler, Lillian Maria Araújo de Rezende Alvares, Eduardo Amadeu Dutra Moresi, Ana Paula Bernardi da Silva

As contribuições da inteligência competitiva para a estratégia no contexto de uma instituição bancária

FULD, L. The new competitor intelligence. New York: John Wiley \& Sons, 1994.

GANESH, U.; MIREE, C. E.; PRESCOTT, J. Competitive intelligence field research: moving the fiel foward by setting a research agenda. Journal of Competitive Intelligence and Management, v. 1, n. 1, p. 1-14, Spring 2003.

GARCIA, T. D. Seminário de Inteligência Competitiva: informação e conhecimento. México: Innestec, 1997. p.21.

GOMES, E.; BRAGA, F. Inteligência competitiva no Brasil: uma realidade corporativa. Revista Puzzle, ano 6, n. 23, 2006.

HEPPES, D.; DU TOIT, A. Level of maturity of the competitive intelligence function: case study of a retail bank in South Africa. In: ASLIB Proceedings. United Kingdom: Emerald Group Publishing Limited, 2009. p. 48-66.

LEÃO, P. R. C. et al. A relevância da metodologia de cenários para instituições financeiras em momentos de crise. Future Studies Research Journal: Trends and Strategies, v 2, n. 2, p. 38-55, 2010.

MARCIAL, E. C. Aspectos fundamentais da inteligência competitiva e a ciência da informação. 2013. 252 f., il. Tese (Doutorado em Ciência da Informação). Universidade de Brasília, Brasília, 2013.

MARIN, J.; POULTER, A. Dissemination of competitive intelligence. Journal of Information Science, 2004. Disponível em:

$<$ http://jis.sagepub.com/content/30/2/165.short 2. Acesso em: 04 abr. 2014.

MENDES, A.; MARCIAL, E.; FERNANDES, F. Fundamentos da Inteligência competitiva. v. 1. Coleção Inteligência Competitiva. Brasília: Thesaurus Editora, 2010.

MILLÁN, J. T.; COMAI, A. La inteligência competitiva em las multinacionales catalanas. El Professional de La información, Barcelona, v. 13, n. 6, p. 478480, 2004.

MORESI, E. A. D. Inteligência organizacional: um referencial integrado.

Ciência da Informação, v. 30, n. 2, p.35-46, ago. 2001.

OLIVEIRA, P. H. Eficiência em inteligência competitiva no contexto das organizações brasileiras: uma abordagem pela VBR e DEA. 2013. 286 f., il. Tese (Doutorado em Administração) - Universidade Federal de Minas Gerais, Belo Horizonte, 2013. 
Maíra Esteves de Carvalho, Helga Cristina Hedler, Lillian Maria Araújo de Rezende Alvares, Eduardo Amadeu Dutra Moresi, Ana Paula Bernardi da Silva

As contribuições da inteligência competitiva para a estratégia no contexto de uma instituição bancária

OLIVEIRA, O.; FORTE, S. A indústria bancária brasileira: Construindo cenários prospectivos e identificando as estratégias de utilização mais provável. Revista Portuguesa e Brasileira de Gestão, v 8, n. 2, p. 64-77, 2009.

POPA, I. A; CUCUI, G. A Framework for Enhancing Competitive Intelligence Capabilities using Decision Support System based on Web Mining Techniques. Int. J. of Computers, Communications \& Control, n. 4, p. 326-334, 2009.

PRESCOTT, J. E., MILLER, S. H. Inteligência competitiva na prática: técnicas e práticas bem sucedidas para conquistar mercados. Rio de Janeiro: Editora Campos, 2002. 371p.

SANTOS, R. N. M. Métodos e ferramentas para gestão de inteligência e do conhecimento. Perspectivas em Ciência da Informação, Belo Horizonte, v 5, n. 2, p. 205-215, jul./dez. 2000.

STRATEGIC and Competitive Intelligence Professionals - SCIP. Disponível em: <http://www.scip.org >. Acesso em: 15 mar. 2014.

TARAPANOFF, K. Inteligência, Informação e Conhecimento. Brasília: IBICT; UNESCO, 2006.

TERRA, J. C. C. Gestão do conhecimento e inteligência competitiva. 2008. Disponível em:

$<$ http://www.terraforum.com.br/biblioteca/Documents/Gesta0\%20do\%20Conhec imento\%20e\%20Inteligencia\%20Competitiva.pdf>. Acesso em: 12 abr. 2014.

TRINDADE, A.B.; REBELO, L.M.B. Minimizando riscos na gestão estratégica: proposta de uso de inteligência competitiva em instituições de ensino e pesquisa. In: ABRAIC: Prêmio de Inovação em Inteligência Competitiva. Brasília: ABRAIC / FINEP, 2005. p.47-67.

TRZECIAK, D. S.; SCHENATTO, F. J. A.; ABREU, A. F. Inovação e inteligência competitiva: uma abordagem integradora sob o enfoque dos processos. In: ENCONTRO NACIONAL DE ENGENHARIA DE PRODUÇÃO, 28., 2008, Rio de Janeiro. Anais... Rio de Janeiro: ABERPRO, 2008.

VIDIGAL, F.; NASSIF, M. E. Inteligência Competitiva: metodologias aplicadas em empresas brasileiras. Informação \& Informação, Londrina, v. 17, n. 1, p. 93-119, 2012. 


\title{
THE CONTRIBUTIONS OF COMPETITIVE INTELLIGENCE FOR STRATEGY IN THE CONTEXT OF A BANK
}

\begin{abstract}
Introduction: The Brazilian banking have generally similar products and services, with little distinction between them. Therefore, the use of strategic and intelligent way information can determine the success and permanence in the market. Competitive intelligence $(\mathrm{Cl})$ have been concerned about the management of this information, structuring their process to improve the knowledge of its competitors, anticipating in order to conquer new markets and achieve better results. Objective: To understand how the IC has contributed to the achievement of strategic objectives of a bank from the perception of managers, addressing the structure, personnel and technological resources of the IC. Methodology: By systematically reviewing scientific literature concerning IC processes, the research procedures along with its specific analytical tools, have been defined. For the current study, the answers given by managers, working for the abovementioned sector, to a close-ending interview have been taken into account. Out of overall 50 employees from strategic, tactical and operational levels, 15 employees have been included in the final corpus, which consists in interviews and focus-groups. The results were split into four categories (vision, cycle, practices and challenges) where each includes its themes. Results: The $\mathrm{Cl}$ was considered an essential tool to be implemented in the strategic process although presente in early stage. However, already needs to show if there were a possible correlation between the intelligence process and our results, which should allow to improve its strategic value. Conclusions: Theoretically, this study contributes to understanding of $\mathrm{Cl}$ processes by considering a specific scientific approach, which defines the dynamic cycle of these processes. From a pragmatic point of view, otherwise, na in-depth implementation and redefinition of the $\mathrm{Cl}$ processes has been promoted.
\end{abstract}

Descriptors: Competitive Intelligence. Decision Making. Strategy.

\section{LAS CONTRIBUCIONES DE LA INTELIGENCIA COMPETITIVA PARA LA ESTRATÉGIA EN EL CONTEXTO DE UMA INSTITUCION BANCÁRIA}

\begin{abstract}
RESUMEN
Introducción: Las instituciones bancarias en el Brasil tienen, en general, productos y servicios muy parecidos con pocas diferencias entre si. De esta manera, el uso de la información estratégica e inteligente puede determinar el éxito y la permanencia en el mercado. La inteligencia competitiva (IC) se han preocupado por la gestión de esta información, la estructuración de su proceso de mejorar el conocimiento de sus competidores, anticipándose, a fin de conquistar nuevos mercados y lograr mejores
\end{abstract}


Maíra Esteves de Carvalho, Helga Cristina Hedler, Lillian Maria Araújo de Rezende Alvares, Eduardo Amadeu Dutra Moresi, Ana Paula Bernardi da Silva

As contribuições da inteligência competitiva para a estratégia no contexto de uma instituição bancária

resultados. Objetivo: Comprender como la IC ha contribuido para el alcance de los objetivos estratégicos de un Banco a partir de la percepción de los gerentes, dirigiéndose a la estructura, el personal y los recursos tecnológicos de la IC. Metodología: La caracterización de la visión sistémica del proceso de $\mathrm{Cl}$ en las instituciones financieras se llevó a cabo a través de la literatura, que culminó en la preparación de herramientas de búsqueda. Esto se aplica a los gerentes de banco de grande porte. Quince de un total de cincuenta empleados de los niveles estratégicos, tácticos y operativos del IC participaron en la fase de recopilación de datos, que incluye entrevistas y un grupo de enfoque. Los resultados se agruparon en cuatro categorías (visión, ciclo, prácticas y desafíos), donde cada uno incluye sus temas. Resultados: La IC se consideraba un elemento clave en el proceso estratégico, aunque todavía presente en etapa temprana. Un desafío clave es relacionar los esfuerzos de inteligencia con los resultados reales, valorando así su valor estratégico. Conclusiones: Como un aporte teórico de este trabajo se ha propuesto un proceso de IC, que incluye diferentes enfoques científicos, que resumen la dinámica de los pasos en el camino. La contribución práctica es a través de la implementación y estructuración del proceso de IC en detalle.

Descriptores: Competitiva Inteligencia. Toma de Decisiones. Estratégica. 